\title{
Dynamical Dipole and Equation of State in N/Z Asymmetric Fusion Reactions
}

\author{
Agnese Giaz ${ }^{1 \mathrm{a}}$, Anna Corsi ${ }^{1}$, Franco Camera ${ }^{1}$, Oliver Wieland ${ }^{2}$, Vladimir L.Kravchuk ${ }^{3}$, Sandro \\ Barlini $^{4}$, Rosa Alba ${ }^{5}$, P.Bednarczyk ${ }^{6}$, Angela Bracco ${ }^{1}$, Giorgio Baiocco ${ }^{7}$, Luigi Bardelli ${ }^{4}$, Giovanna \\ Benzoni ${ }^{2}$, M.Bini ${ }^{4}$, Nives Blasi ${ }^{2}$, Sergio Brambilla ${ }^{2}$, Mauro Bruno ${ }^{7}$, Giovanni Casini ${ }^{4}$, Michal \\ Ciemala $^{6}$, Marco Cinausero ${ }^{3}$, M.Chiari ${ }^{4}$, Maria Colonna ${ }^{9}$, Fabio Celso Luigi Crespi ${ }^{1}$, Michela \\ D’Agostino $^{7}$, Meltem Degerlier ${ }^{3}$, Massimo Di Toro ${ }^{9}$, Fabiana Gramegna ${ }^{3}$, Maria Kmiecik ${ }^{6}$, Silvia \\ Leoni $^{1}$, C.Maiolino ${ }^{5}$, Adam Maj ${ }^{4}$, Tommaso Marchi ${ }^{3}$, K.Mazurek ${ }^{6}$, W. Meczynski ${ }^{6}$, Benedicte \\ Million ${ }^{2}$, Daniele Montanari ${ }^{1}$, Luca Morelli ${ }^{5}$, Adriana Nannini ${ }^{4}$, Roberto Nicolini ${ }^{1}$, G.Pasquali ${ }^{4}$, \\ S.Piantelli ${ }^{4}$, A.Ordine ${ }^{8}$, Giacomo Poggi ${ }^{4}$, V.Rizzi $^{3}$, Carmelo Rizzo $^{9}$, Domenico Santonocito ${ }^{5}$, \\ ValeriaVandone $^{1}$, G.Vannini ${ }^{5}$ \\ ${ }^{1}$ Dipartimento di Fisica, Università di Milano and INFN sezione di Milano, via Celoria 16, 20133 \\ Milano, Italy \\ ${ }^{2}$ INFN sezione di. Milano, via Celoria 16, 20133 Milano, Italy \\ ${ }^{3}$ Istituto Nazionale di Fisica Nucleare, Laboratori Nazionali di Legnaro, Legnaro, Italy \\ ${ }^{4}$ Dipartimento di Fisica, Università di Firenze and INFN sezione di Firenze, Firenze, Italy \\ ${ }^{5}$ Istituto Nazionale di Fisica Nucleare, Laboratori Nazionali del Sud, Catania, Italy \\ ${ }^{6}$ The Henryk Niewodniczański Institute of Nuclear Physics, PAN, 31-342,Krakow, Poland \\ ${ }^{7}$ Dipartimento di Fisica, Università di Bologna and INFN sezione di Bologna, Bologna, Italy \\ ${ }^{8}$ INFN sezione di Napoli, Napoli, Italy \\ ${ }^{9}$ Dipartimento di Fisica e Astronomia dell'Università di Catania and Istituto Nazionale di Fisica \\ Nucleare, Laboratori Nazionali del Sud, Catania, Italy
}

\begin{abstract}
In heavy ion reactions, in the case of $\mathrm{N} / \mathrm{Z}$ asymmetry between projectile and target, the process leading to complete fusion is expected to produce pre-equilibrium dipole $\gamma$-ray emission. It is generated during the charge equilibration process and it is known as Dynamical Dipole. A new measurement of the dynamical dipole emission was performed by studying ${ }^{16} \mathrm{O}+{ }^{116} \mathrm{Sn}$ at $12 \mathrm{MeV} / \mathrm{u}$. These data, together with those measured at $8.1 \mathrm{MeV} / \mathrm{u}$ and $15.6 \mathrm{MeV} / \mathrm{u}$ for the same reaction, provide the dependence on the Dynamical Dipole total emission yield with beam energy and they can be compared with theoretical expectations. The experimental results show a weak increase of the Dynamical Dipole total yield with beam energies and are in agreement with the prediction of a theoretical model based on the Boltzmann-Nordheim-Vlasov (BNV) approach. The measured trend with beam energy does not confirm the rise and fall behavior previously reported for the same fused compound but with a much higher dipole moment.
\end{abstract}

\section{Introduction}

\footnotetext{
${ }^{\text {a } C o r r e s p o n d i n g ~ a u t h o r: ~ a g n e s e . g i a z @ m i . i n f n . i t ~}$
} 
The process leading to complete fusion is expected to produce pre-equilibrium gamma-ray emission if an N/Z asymmetry between the projectile and the target is present. In such a situation, a time dependent dipole moment, which is associated with pre-equilibrium $\gamma$-radiation emission, is formed. This phenomenon, known as Dynamical Dipole (DD), is expected to depend on the energy of the projectile and on the size of the dipole moment. An aspect which can be studied using stable beams is the DD dependence on the beam energy [1-3], whereas the study of the DD dependence on the dipole moment requires large N/Z ratio and, therefore, exotic beams.

The existing experimental data are still rather scarce and, although some systematic exists in the $\mathrm{A}=132$ mass region, the experimental data do not seem to follow the theoretical predictions concerning the dependence of the DD yield emission on beam energy. Experimentally, it was observed that Dynamical Dipole emission increases with beam energy up to $\approx 10 \mathrm{MeV} / \mathrm{u}$, then the yield significantly decreases. In this work the DD yield at $12 \mathrm{MeV} / \mathrm{u}$ for the ${ }^{16} \mathrm{O}+{ }^{116} \mathrm{Sn}$ reaction will be discussed. These data are combined with those of ref [1] where the DD total yield was studied in the same reaction but at different beam energies.

The model which better describes the Dynamical Dipole uses the dynamical evolution of the dipole in the framework of BNV (Boltzmann Nordheim Vlasov) prescription and employs the bremsstrahlung expression for the calculation of the photon yield. It is important to point out that the model critically depends on the nuclear equation of state with its symmetry term and on its density dependence. In ${ }^{16} \mathrm{O}+{ }^{116} \mathrm{Sn}$ the dependence on the nuclear equation of state (EOS) is not extremely evident although some sensitivity can be achieved by selecting small values of the impact parameter. The theoretical model predicts that the dependence on the symmetry term of EOS will be evident using exotic nuclei (i.e. ${ }^{132} \mathrm{Sn}$ ) as projectiles [4].

\section{Experimental set up}

The experiment was performed in Laboratori Nazionali di Legnaro by using the GARFIELD HECTOR apparatus. The HECTOR array [5] consists of eight large volume (about $14 \times 18 \mathrm{~cm}$ ) $\mathrm{BaF}_{2}$ crystals for the detection of high-energy $\gamma$ rays. The GARFIELD (General ARray for Fragment Identification and for Emitted Light particles in Dissipative collision) array [6] measures light charged particles. It consists of $\Delta \mathrm{E}-\mathrm{E}$ gaseous micro strip and $\mathrm{CsI}(\mathrm{Tl})$ scintillation detectors lodged in the same gas volume. The evaporated residues are detected in a wall of 32 PHOSWICH detectors, from FIASCO array [7], placed at forward angles.

\section{Results}

The statistical gamma decay of the giant dipole resonance (GDR) and the DD pre-equilibrium emissions have the same dipolar nature and appear in the same energy window (10-20 MeV). To separate the two contributions it is necessary to compare the high-energy gamma-rays spectra measured in an 'asymmetric' fusion-evaporation reaction (where the DD emission is present) and a 'symmetric' one (where the DD emission is not present-). The experimental signature of the DD emission is an excess of counts in the energy region of 10-20 MeV with respect to the statistical gamma decay of the GDR, as for example shown in left panel of Figure 1.

In section 3.1, the data analysis and the results at $12 \mathrm{MeV} / \mathrm{u}$ will be discussed and compared with the data acquired in the previous GARFIELD - HECTOR campaign and reported in [1]. The theoretical predictions for the DD yield will be compared with the experimental data in section 3.2. In section 3.3 the measured angular distribution will be presented.

\subsection{Experimental results}

As already mentioned in the introduction, the DD total yield was extracted for the reaction ${ }^{16} \mathrm{O}+{ }^{116} \mathrm{Sn}$ $={ }^{132} \mathrm{Ce}^{*}$ at $12.1 \mathrm{MeV} / \mathrm{u}$ by the measurements of the high-energy gamma-ray spectrum gated by the 
evaporation residues and prompt gamma-rays. The reference 'symmetric' high-energy gamma-rays spectrum was represented by the spectrum measured in the reaction ${ }^{64} \mathrm{Ni}+{ }^{68} \mathrm{Zn}={ }^{132} \mathrm{Ce}^{*}$ studied in ref [1]. The analysis of the 'symmetric' reaction provides the statistical model and the GDR parameters then used to extract the DD total yield from the high-energy gamma-rays spectrum measured in the 'asymmetric' reaction, namely ${ }^{16} \mathrm{O}+{ }^{116} \mathrm{Sn}={ }^{132} \mathrm{Ce}^{*}$ at $12.1 \mathrm{MeV} / \mathrm{u}$.

The excitation energy of ${ }^{132} \mathrm{Ce}$ compound nucleus is a second important observables which has to be evaluated through the measurement of the particle pre-equilibrium energy loss in GARFIELD. It is important to point out that the pre-equilibrium particle energy loss is a critical parameters as the total DD yield almost linearly depends on it. Once the temperature of the thermalized ${ }^{132} \mathrm{Ce}$ compound nucleus is extracted and the GDR statistical model parameter known, it is possible to extract the DD total yield by comparing the measured gamma ray spectrum in the reaction ${ }^{16} \mathrm{O}+{ }^{116} \mathrm{Sn}={ }^{132} \mathrm{Ce}^{*}$ with the prediction of the statistical model (see left panel of figure 1). The measured DD total yield shows a weak rising trend as a function of the beam energy as shown in right panel of Figure 1.
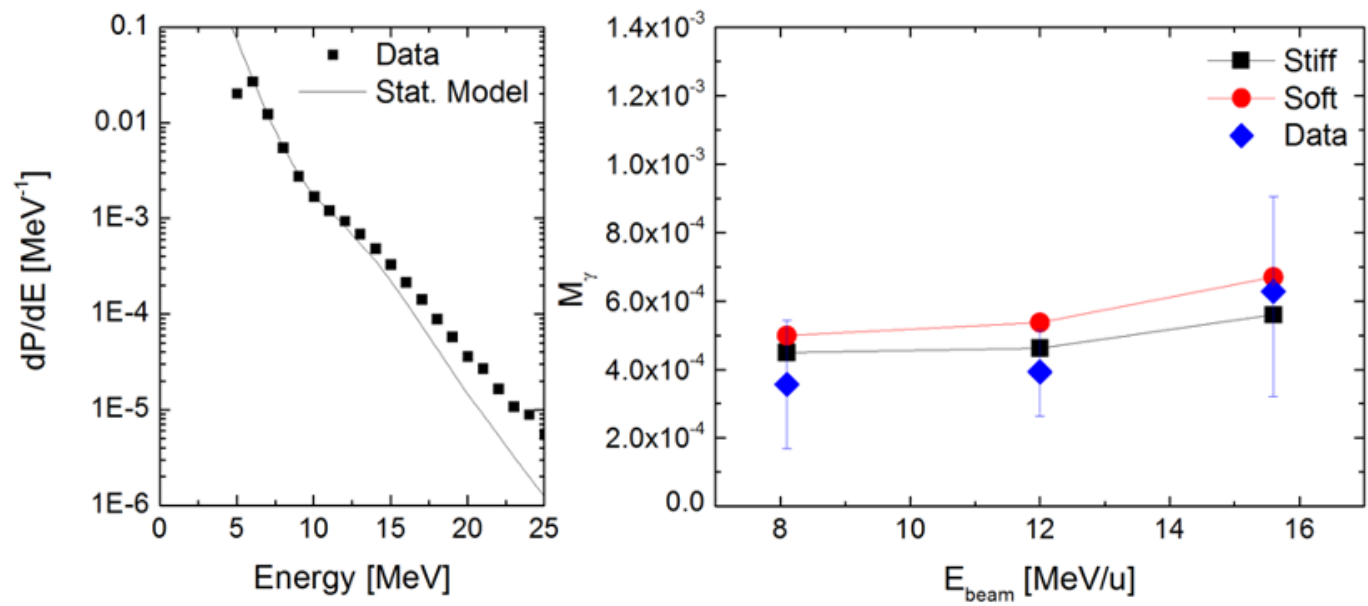

Figure 1. Left Panel: The high-energy gamma-rays spectrum measured in the reaction ${ }^{16} \mathrm{O}+{ }^{116} \mathrm{Sn}={ }^{132} \mathrm{Ce}^{*}$ at 12.1 MeV/u compared with the statistical model calculations. Right Panel: the DD yield as a function of the beam energy. The experimental data (diamonds) are compared with the theoretical prediction of the BNV model, for both the parameterizations of the symmetry term of the EOS. The asy-stiff parameterization is shown by squares, instead the asy-soft one with circles.

\subsection{Theoretical prediction}

The theoretical estimation of the amount of pre-equilibrium $\gamma$ emission was calculated by using the BNV model $[4,9]$. This is achieved by considering the evolution of the collective dipole acceleration from the 'contact' time (when it suddenly rises) until it is completely damped to a pure "thermal" component. In this way it is possible to consistently calculate the whole contribution of the preequilibrium dipole radiation to the photon yield. Using this model, we simulated the fusion dynamic for the system ${ }^{16} \mathrm{O}+{ }^{116} \mathrm{Sn}$ at $8.1,12$ and $15.6 \mathrm{MeV} / \mathrm{u}$.

The BNV simulations were performed for different impact parameters, as shown in Figure 2. The total DD yield has been obtained from a weighted averaged on the different impact parameters. It is interesting to point out that the differences between asy-stiff and asy-soft parameterization are larger at small value of the impact parameter. In the case low spin could be selected (namely selecting a small impact parameter 'b') a quite large sensitivity to EOS (20\%) would be achieved also in the present experiment, as shown in Figure 2.

The BNV model predictions show a rising trend as a function of the beam energy similar to the one found in the the experimental data. The results of the BNV simulations compared with the experimental data are shown in the right panel of Figure 1. 


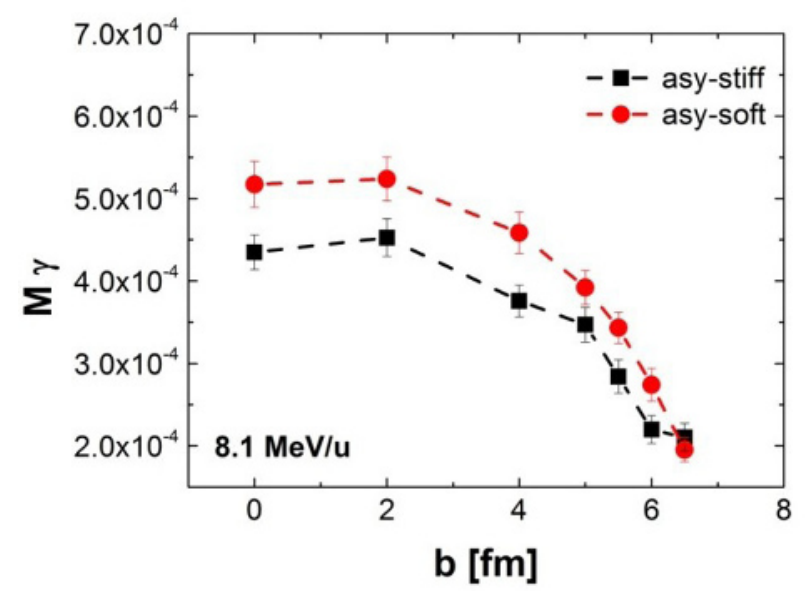

Figure 2. The trend of the DD yield as a function of the impact parameter. Squares are for the asy-stiff parameterization of EOS, instead circles are for the asy-soft one.

\subsection{Angular distribution}

The experimental data permit, additionally, to extract the DD angular distribution. The angular distribution should have a dipolar character but due to the rotation of the dipolar axis it is expected to be quenched with respect to a pure dipolar one. In literature, it is possible to find two different measurements for the DD dipole angular distribution. The one reported in [3] is consistent with an emission from a pure dipole oscillating along the beam axis while the angular distribution reported in [1] shows a strong quenching as expected from the rotation of the dipolar axis. The measured angular distribution at $12 \mathrm{MeV} / \mathrm{u}$ shows a quenching factor similar to the one of ref [1]. However, BNV predicts a quenching factor which is much larger than the measured one.

\section{Conclusion}

The DD $\gamma$-ray yield for ${ }^{16} \mathrm{O}+{ }^{116} \mathrm{Sn}$ at $12 \mathrm{MeV} / \mathrm{u}$ was extracted and compared with BNV theoretical calculations. Theory reproduces the measured behavior of the experimental total DD yield. A large (even though quenched) angular distribution has been measured if compared to theoretical calculations. A sensitivity to EOS was found at small impact parameters event using stable beams $\left({ }^{16} \mathrm{O}+{ }^{116} \mathrm{Sn}\right)$.

\section{References}

1. A. Corsi, et al, Phys. Lett. B, 679, 197 (2009).

2. D. Pierroutsakou, et al, Phys. Rev. C, 80, 024612 (2009).

3. B. Martin, et al, Phys. Lett. B, 664, 47 (2008).

4. V. Baran, et al, Phys. Rev. C, 79, 021603 (2009).

5. A. Maj, et al, Nucl. Phys. A, 571, 185 (1994).

6. F. Gramegna, et al, Nucl. Instr. Meth. A, 389, 474 (1997).

7. M.Bini, et al, Nucl. Instr. Meth. A, 515, 497 (2003).

8. O. Wieland, et al, Phys. Rev. Lett., 97, 012501 (2006).

9. V. Baran, et al, Phys. Rev. Lett., 87182501 (2001). 